\title{
Il problema dell'alimentazione durante la prima guerra mondiale
}

\author{
Studio di caso \\ a cura di Nadia Olivieri \\ Istituto veronese per la storia della Resistenza e dell'età contemporanea
}

\begin{abstract}
L'approvvigionamento alimentare della popolazione e delle truppe al fronte fu uno dei problemi che gli stati coinvolti nel primo conflitto mondiale si trovarono a dover gestire, con forme di intervento diretto nella produzione e distribuzione delle risorse, ma anche tramite azioni di propaganda rivolte alla popolazione, chiamata a limitare i consumi per sostenere lo sforzo bellico. Lo studio di caso qui proposto permette di indagare alcuni aspetti di questa tematica, che vanno dai problemi di sostituzione della manodopera maschile nelle campagne e di assenza di animali da lavoro a seguito delle requisizioni alle restrizioni alimentari imposte alla popolazione, dalla propaganda per la limitazione dei consumi al calcolo dell'approvvigionamento calorico delle truppe al fronte.
\end{abstract}

\section{Tags}

Prima guerra mondiale, alimentazione, propaganda, limitazione dei consumi, tessera annonaria, 1914-1918

\section{Indice}

Introduzione

Perché l'alimentazione

Un tema solo apparentemente semplice

Il cibo e la grande guerra

Il lavoro di preparazione

Due distinte versioni

La sequenza didattica

Bibliografia

Sitografia

Lo studio di caso

Testo iniziale

Dossier di documenti

Dossier di lavoro

\section{Perché l'alimentazione}

Nel prospettare la progettazione di un'attività laboratoriale in classe, il tema dell'alimentazione è fra i più scelti dagli insegnanti: rispondendo ad uno dei bisogni fondamentali dell'uomo, il cibo viene ritenuto argomento di per sé interessante e coinvolgente per gli alunni sin dalla scuola primaria. Non a caso, nell'affrontare lo studio delle civiltà del passato ampio spazio viene dato 
all'alimentazione ${ }^{1}$ e alla sua connessione con le attività economiche, tanto produttive quanto commerciali.

\section{Un tema solo apparentemente semplice}

In realtà quello dell'alimentazione è un tema più complesso di quanto generalmente inteso. "I menù, le ricette, le liste del vasellame o delle cibarie parlano da sé e nello stesso tempo sono assolutamente oscuri, mescolando nomi di piatti e di derrate attuali, se non familiari, ad altri indecifrabili che la tradizione lessicografica italiana, nemica della cucina e vergognosa della vita materiale, non si è mai peritata di registrare ${ }^{2}{ }^{2}$, ci ricordano gli autori nell'introduzione all'Annale della Storia d'Italia Einaudi interamente dedicato all'alimentazione. E se è vero che a mano a mano che dai tempi antichi ci si avvicina alla contemporaneità - e tanto più se ci si concentra sullo spazio geografico nazionale - questa avvertenza diviene sempre meno rilevante, è innegabile che la storia dell'alimentazione si rivela "una prospettiva d'indagine utile a cogliere, dall'angolatura specifica delle sue variabili, i mutamenti profondi della società [...]. II cibo infatti rappresenta, ad un tempo, una spia significativa ed una conseguenza del complesso intreccio tra continuità e trasformazione che ha attraversato i processi di modernizzazione delle società industriali $»^{3}$.

\section{Il cibo e la grande guerra}

La prima guerra mondiale fu, da questo punto di vista, un momento di svolta determinante, sia per i paesi già industrializzati, sia per quelli, come l'Italia, ancora in via di modernizzazione. L'intervento dello stato in economia divenne ineludibile e si esplicò a tutto tondo, tanto nella regolazione degli ammassi, quanto nell'indirizzo della produzione e nella distribuzione delle risorse. L'intervento si accompagnò ad un'intensa campagna propagandistica rivolta all'intera popolazione, che se da un lato andava sperimentando restrizioni alimentari e un progressivo deterioramento qualitativo del cibo $^{4}$, dall'altra veniva chiamata a dare il proprio contributo allo "sforzo bellico" proprio attraverso la limitazione dei consumi e l'attivazione - ovviamente nelle città - di forme di autoproduzione di beni alimentari. Una "mobilitazione civile" volta a compattare il cosiddetto "fronte interno", che approdò, naturalmente, anche nelle scuole 5 .

\footnotetext{
${ }^{1}$ Infatti, «uno dei convincimenti più diffusi è che l'alimentazione dia concretezza allo studio della civiltà, che i costumi conviviali siano uno specchio della sua esistenza» [Capatti, De Bernardi e Varmi (acd) 1998, XXV].

2 Ibidem.

3 Ibidem.

${ }^{4}$ Già alla fine di marzo del 1915 una grave crisi granaria indusse le autorità a stabilire che il pane venisse confezionato in forme non inferiori a 500 grammi e con farina abburattata in ragione dell' $80 \%$. Questo "pane di guerra" divenne, col passare del tempo, sempre meno gradevole: veniva venduto raffermo e confezionato con surrogati di farina di ogni genere (riso, granoturco, castagne, lupini). A partire dal 1916 iniziarono gli interventi di regolazione dei consumi: vennero introdotti i calmieri (prima per il grano e poi per lo zucchero); si ridusse la distribuzione di cibi e bevande nei locali pubblici; venne limitata la vendita di dolci e di carne (con la chiusura delle macellerie nei giorni di giovedì e venerdi). Nel novembre del 1917 comparve la tessera annonaria: a partire dal $1^{\circ}$ novembre, ogni cittadino aveva quotidianamente diritto a 250 grammi di pane, 90 grammi di pasta e 40 di riso. In seguito, il tesseramento si estese a carne bovina, olio, grassi animali, burro, formaggi. Le marcate restrizioni alimentari e il deterioramento qualitativo del cibo influirono negativamente sulle condizioni di vita degli italiani [Dentoni 2014, 230-238].

${ }^{5}$ «Giovani e giovanissimi sono poi chiamati a dare il loro contributo in varie attività di supporto a favore del conflitto: tra le tante, si impegnano soprattutto nella fabbricazione di «scalda-ranci» (rotoli di carta imbevuti di paraffina che, accesi, vengono utilizzati dai soldati in trincea per riscaldare i pasti) e nel dissodamento di terre incolte con l'intento di diffondere il concetto che l'Italia deve essere autosufficiente per l'alimentazione». La citazione è tratta dal sito che I'Istituto Parri di Bologna ha dedicato al tema dell'approvvigionamento alimentare nel Novecento: un esempio
} 
Quello a seguire, è uno studio di caso preparato da alcuni insegnanti veronesi a conclusione di un percorso formativo sulla didattica della storia svolto nell'anno scolastico $2015-2015^{6}$. Gli aspetti che vi vengono trattati sono molteplici: la sostituzione della manodopera maschile nelle campagne, l'assenza di animali da lavoro a seguito delle requisizioni, le restrizioni alimentari imposte alla popolazione, la propaganda per la limitazione dei consumi, l'approvvigionamento calorico delle truppe al fronte. Alcuni dei documenti scelti per lo svolgimento dell'attività in classe si riferiscono al territorio provinciale veronese, ma, qualora si volesse lavorare su altre realtà territoriali, per l'insegnante non sarà difficile, nella molteplicità di studi locali pubblicati in questi anni di celebrazione del centenario, rinvenire materiali idonei al caso e sostituirli a quelli qui proposti.

\section{Due distinte versioni}

Dello studio di caso sono state preparate due distinte versioni: una, che si potrebbe definire "generale", viene presentata in forma estesa, con un nutrito pacchetto di documenti e suggerimenti didattici declinabili a diversi livelli di complessità (vedi allegato 1); la seconda è invece la versione applicata nel percorso didattico proposto dall'Istituto veronese per la storia della Resistenza agli alunni della scuola secondaria di primo grado, con una "dossier di documenti" selezionato fra i diversi disponibili e con un "dossier di lavoro" per i gruppi con gli esercizi già predisposti e pronti all'uso. Una sorta di esempio di come uno studio di caso possa essere ridotto e adattato per l'uso in classe.

La sequenza didattica

Il percorso è stato predisposto in modo tale da esaurirsi in due ore, ricorrendo all'organizzazione in gruppi di apprendimento cooperativo, secondo questa sequenza:

- brevissima introduzione di tipo metodologico-organizzativo sul lavoro da svolgere e costituzione dei gruppi di lavoro (volendo, si può far ricavare l'argomento del laboratorio da fotografie pertinenti l'argomento, che possono servire alla costituzione casuale dei gruppi se tagliate in tre o quattro pezzi e ricomposte dai ragazzi stessi);

- lavoro sullo studio di caso. I gruppi ricevono un pacchetto di materiale comprendente il testo iniziale, il dossier di documenti e il dossier di lavoro. La consegna è quella di leggere insieme il testo iniziale e poi iniziare a svolgere gli esercizi richiesti nel dossier di lavoro. Ciascun gruppo parte da un esercizio diverso (il gruppo 1 dal numero 1, il 2 dal 2 e così via), prosegue poi con gli esercizi successivi (dopo l'esercizio 7 si ricomincia dall'1) fino ad esaurire il tempo concesso, che è di un'ora piena di lavoro. Nel gruppo si possono assegnare i ruoli: 1) il lettore (e rilettore) del testo iniziale; 2) lo scrittore/sottolineatore; 3) il portavoce di gruppo; 4) il responsabile organizzativo (prende i materiali e controlla tono di voce e tempo di lavoro);

- al termine di questa fase, ci si riunisce in plenaria. Ogni gruppo riferisce lo svolgimento dell'esercizio da cui è partito, ma tutti gli altri gruppi che sono arrivati ad affrontare lo stesso

eccellente dei molteplici percorsi che lo studio storico dell'alimentazione può aprire. Sul progetto nel complesso si veda la pagina di presentazione: https://guerrainfame.it/progetto. Nello specifico, la frase citata si trova in https://guerrainfame.it/limitazione consumi scuola/limitazione consumi/tesseramento, nella parte dedicata alla campagna per la limitazione dei consumi a scuola.

${ }^{6}$ Il percorso è stato svolto presso l'Istituto comprensivo di Pescantina 1 e ha coinvolto insegnanti dei diversi ordini di scuola, compresi docenti della scuola dell'infanzia, che hanno ideato un'intera settimana di attività con i bambini più piccoli. Attività che non è possibile esaminare in questa sede, ma che è stata illustrata in varie occasioni di scambio di "buone pratiche" a livello regionale. 
esercizio possono intervenire a integrare e/o correggere il lavoro. In questo modo, indipendentemente dal fatto di aver svolto o meno tutto il dossier (non è detto che, nel tempo concesso, ce la facciano, ma in questo modo ogni gruppo può lavorare secondo i propri ritmi), si arriva ad una condivisione - anche solo, per le parti non svolte, di ascolto del lavoro complessivo.

- alla fine, il docente riepiloga i contenuti scoperti e valorizza il lavoro svolto dai ragazzi.

Lo studio di caso

\section{TESTO INIZIALE}

\section{IL PROBLEMA DELL'ALIMENTAZIONE DURANTE LA PRIMA GUERRA MONDIALE}

Nel 1914 in Europa scoppiò la prima guerra mondiale, che vide scontrarsi Russia, Francia e Inghilterra da un lato, contro Austria-Ungheria e Germania dall'altro. L'Italia decise di entrarvi nel 1915 per conquistare Trento e Trieste, all'epoca ancora austriache. Fu una guerra che investì ogni aspetto della vita dei paesi che vi parteciparono.

All'inizio, tutti i paesi coinvolti erano convinti che la guerra sarebbe durata poco. Per questo, nessun governo si preoccupò di come assicurare l'approvvigionamento dell'esercito e della popolazione nel lungo periodo. Ben presto il conflitto divenne invece una lunga ed estenuante guerra di posizione e l'alimentazione dei soldati e della popolazione divenne un grosso problema.

Neanche I'Italia, entrata in guerra nel giugno del 1915, si era preoccupata di programmare la produzione e il consumo di beni alimentari. Dopo l'ingresso in guerra, la produzione di grano interna iniziò subito a risentire della mancanza di uomini. I soldati provenivano infatti soprattutto dal settore agricolo. In loro assenza, i lavori nelle campagne venivano svolti da donne, vecchi e bambini, spesso senza l'aiuto degli animali da lavoro, che erano stati requisiti dall'Esercito.

Per la popolazione divenne difficile procurarsi il cibo, sia per la scarsità, sia per il prezzo sempre più elevato. In principio il Governo italiano intervenne a regolamentare la qualità delle farine usate per la produzione del pane. In seguito, stabilì leggi sempre più severe per controllare la produzione e il consumo di beni alimentari, specialmente di quelli "di lusso", come lo zucchero e la carne. Dal 1917 si ricorse al razionamento, cioè alla limitazione degli acquisti effettuata attraverso una distribuzione controllata dei generi alimentari, attraverso una apposita tessera (la tessera annonaria).

Contemporaneamente si inaugurò una campagna propagandistica per la limitazione dei consumi. Appositi "Comitati" furono istituiti in tutte le città, con lo scopo di tenere conferenze alla popolazione e spiegare come "viver bene mangiando poco". L'opera di propaganda interessò anche le scuole, dove ai ragazzi veniva spiegata l'utilità di coltivare un piccolo orto o di allevare conigli.

La guerra, dunque, non fu un affare riservato ai soli soldati al fronte, ma tutta la nazione venne chiamata a sostenere lo sforzo bellico e il cibo quotidiano fu uno dei problemi che più assillò sia $i$ soldati al fronte, che la popolazione civile. Proprio l'impossibilità di procurarsi i beni alimentari fu uno dei fattori che contribuirono alla sconfitta della Germania. 


\section{DOSSIER DEI DOCUMENTI}

\section{Documento 1}

Fin dai primi giorni di guerra, Verona assume l'aspetto di una città di retrovia [...] Nella vita di tutti i giorni, i sacrifici più pesanti riguardano l'alimentazione ed in particolare il pane, che ne costituisce la base. La vicenda delle disposizioni e dei controlli con cui si cerca di disciplinarne il consumo costituisce una storia nella storia. Ne ricordiamo qui solo qualche passaggio, anche perché il problema non comincia con la guerra, visto che nell'agosto del 1914, quando l'Italia è neutrale, i giornali annunciano che l'amministrazione comunale metterà in vendita alcuni quantitativi di pane a prezzo speciale "per venire incontro ai bisogni della popolazione meno agiata" ("L'Adige", 27 agosto 1914).

Nel 1915, tutta la popolazione, e non solo quella parte eufemisticamente definita "non agiata", si deve accontentare di poco gradevoli pagnotte simili a quelle militari. Nel 1917, va peggio: si vende solo pane raffermo, che a quanto assicura "L'Arena" del 4 gennaio, "sazia più presto" e costituisce perciò "una notevole economia". Ed anche chi pensa di consolarsi con la polenta, viene puntualmente disilluso dal tesseramento, che, sempre nel 1917, fissa in 350 grammi la razione quotidiana per persona.

Anche la carne bovina diviene presto merce rara. La necessità di rifornire l'esercito costringe i civili a ridurre i consumi. Crescono naturalmente i prezzi, con ricadute pesanti su tutto il settore: gli allevatori, attratti dalla possibilità di altissimi guadagni, mandano al macello anche animali destinati alla produzione di latte ed al lavoro. Si cerca perciò di limitarne il consumo e si confida nell'importazione della carne congelata proveniente dall'Argentina. Nel 1916, i giornali scrivono che solo in questo modo si potranno "mantenere le truppe con ottima carne esotica", senza penalizzare oltre la popolazione civile. Nel marzo del 1918, la carne sparisce dai ristoranti per due giorni alla settimana. Un analogo provvedimento era stato preso l'anno prima, con la precisazione che il divieto riguardava anche "salsicce, zamponi e cotechini".

Lo zucchero è oggetto di innumerevoli polemiche e di una ricerca spasmodica da parte dei consumatori. Nel 1916, il consumo viene sottoposto al controllo dello Stato, mentre sono naturalmente soggetti a limitazioni e divieti i dolci. In quello stesso anno, si apre alla Gran Guardia uno spaccio comunale: lo zucchero viene venduto in cartoni da tre etti e non se ne possono acquistare più di due. Si teme infatti la corsa all'accaparramento, ma poi le cose vanno diversamente perché, come sottolineano i giornali, "i consumatori si lamentano per la pessima qualità dello zucchero". L'anno dopo, in uno dei tanti momenti in cui risulta introvabile, il Comune, ne mette in vendita un certo quantitativo nel cortile del Tribunale. La ressa provoca code interminabili, con discussioni così accese, da esigere l'intervento della polizia (“L'Arena", 9 e 10 febbraio 1917) [...]

Testo tratto da Luciani E. 2002, Giornalisti in trincea. L'informazione durante la Grande Guerra in una città di retrovia, Verona: Gemma Editco. 


\section{Documento 2}

\section{Castagnevizza 3/3/'917}

Carissima moglie,

Ti do le mie buone notizie e di te e tutti quanti spero altrettanto. Come già avrai inteso ho ricevuto il Vaglia di f. 20 mentre io intendevo che tu ne trattenessi una metà, ma almeno cinque lire. Di ciò non voglio rimproverarti, anzi così conosco la tua premura, ma d'ora in avanti ti prego fare come ti dico: ora in tasca tengo 33 lire, e durante il riposo che durerà un mese avrò ancora 16 lire, perché come sai ora che sono zappatore effettivo prendo 55 cent. al giorno. Dunque tutti questi denari durante il riposo non faccio tempo a spenderli, perché come ti dissi mi dispiace spenderli così male essendo che tutto costa all'eccesso. Dalla tua lettera speditami il 24/2 intesi pure che le cose vanno sempre più male anche per i borghesi, mi dici che zucchero si stenta trovarne, questo lo credo, tutti quelli che vengono dalla licenza e che stanno in città dicono che non possono più vivere, queste cose fanno male e forse sarà bene perché la guerra potrà finire più presto. Mi dispiace perché so che il latte a te ti piace un poco e ritengo che sarà il companatico della mattina, e a Franceschino poverino che li piace tanto la roba dolce. I denari che terrai per conto tuo non intendo che tu li tieni nell'armadio ma bensì che li spendi per tutti e due, e così ti puoi comperare dei biscotti che costano 40 cent al pacchetto e sono così buoni tanto nel latte come nel vino, e poi compera quello che ti pare, ti raccomando [...] coll'aiuto di Dio ci siamo ancora tutte e tre sani spero almeno. Dunque se continuano così ci avvicineremo alla fine di questa misera vita. [...] Dici di mandarmi un pacchetto di roba da mangiare per questo fa come ti pare certo che mi fai piacere ma voglio che quello che fai lo devi dividere e mangiarne anche voi altrimenti mi fai dispiacere. [...] Vi penso sempre nelle faccende campestri, perché quest'anno dev'essere un grande imbroglio e tu fa quello che puoi e va d'accordo coll'Angelina di trovare almeno delle donne a lavorare. Altro non ti dico, siamo bene intesi solo ti mando tanti baci che tu li ripeterai al nostro caro bambino. Abbi pazienza nel leggere puoi credere ho scritto sui ginocchi stando seduto per terra.

Aff.si saluti tuo aff.mo Marito Benedetto ciao

(lettera che Benedetto Tambara scrisse dal fronte alla moglie Toscana Zambonini, riportata nel libro di Beltrame Menini L. 2014, Ta-pum. Lettere dal fronte, Padova: F.lli Corradin Editori, pp. 187-188) 


\section{Documento 3}

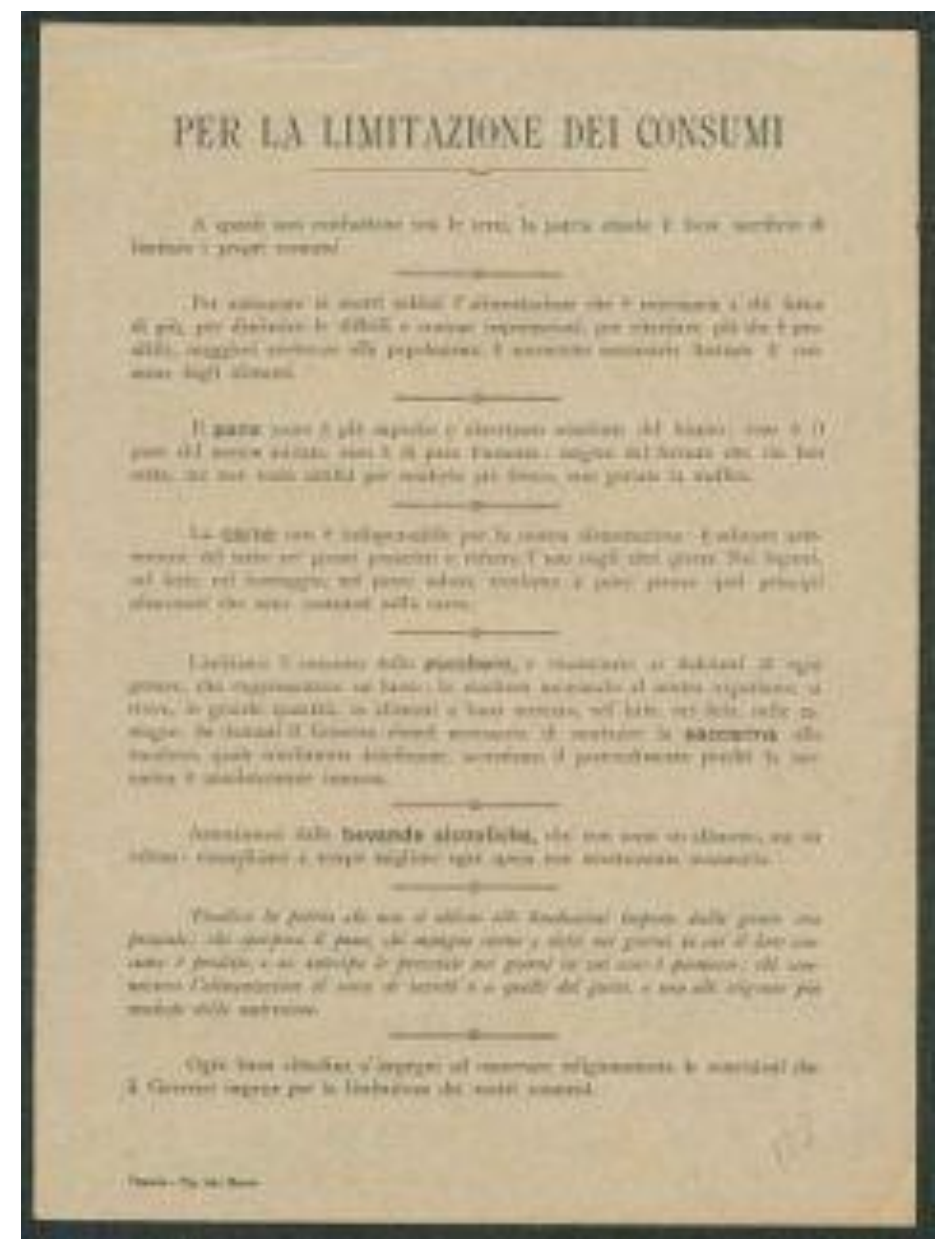

Titolo:

Per la limitazione nei consumi Pubblicazione:

Venezia : Tip. San Marco, [1917]

Descrizione fisica:

1 foglio : litografia, $\mathrm{b} / \mathrm{n} ; 27 \times 20 \mathrm{~cm}$

Note:

Abstract:

Foglio composto di solo testo

Il foglio informa che "Per assicurare ai nostri soldati l'alimentazione che è necessaria a chi fatica di più, per diminuire le difficili e costose importazioni, per ritardare, più che è possibile, maggiori strettezze alla popolazione, è soprattutto necessario limitare il consumo degli alimenti" in particolar modo pane, carne, zucchero, bevande alcooliche.

Ente di appartenenza:

Biblioteca Universitaria Alessandrina

\section{Comitati Provinciali di propaganda per la limitazione dei consumi}

È stata inviata ai Prefetti la seguente circolare:

II Governo del Re è stato obbligato dalla necessità della guerra [...] a stabilire [...] alcune limitazioni e norme nei consumi le quali in verità si risolvono in qualche lieve disagio per le nostre popolazioni. $\mathrm{Ma}$, poiché è intuitivo che le norme legali non bastano ove non le assista e non vigili la coscienza popolare, e d'altro lato non è escluso, e può anzi ritenersi come probabile, che nuove, più severe limitazioni abbiano ad apportarsi in questo campo, il Governo si è convinto della opportunità [...] di iniziare e perseguire [...] un'opera di propaganda [...] nei modi che più sono atti a raggiungere la coscienza delle diverse classi sociali.

[...] poiché il primo compito cui ci proponiamo dedicarci è quello di una intensa propaganda circa la necessità patriottica e i benefici generali ed individuali che deriveranno da una limitazione nei consumi, dovrà il Comitato curare la diffusione degli opuscoli che noi invieremo e vigilare perché essi penetrino in quegli ambienti nei quali la propaganda è più necessaria e può riuscire più efficace. In questi opuscoli noi verremo man mano esponendo le ragioni che determinarono e determineranno i provvedimenti governativi, prospetteremo le ragioni patriottiche ed igieniche che queste limitazioni consigliano; dimostreremo il beneficio che anche per l'avvenire il nostro paese può trarre da una misurata politica annonaria; renderemo chiaro come e perché una norma di vita misurata e frugale possa giovare al più rapido risorgimento economico del paese. 
(articolo pubblicato sul giornale di Verona, "L'Arena”, mercoledì 24 gennaio 1917, p. 3)

\section{Documento 4}

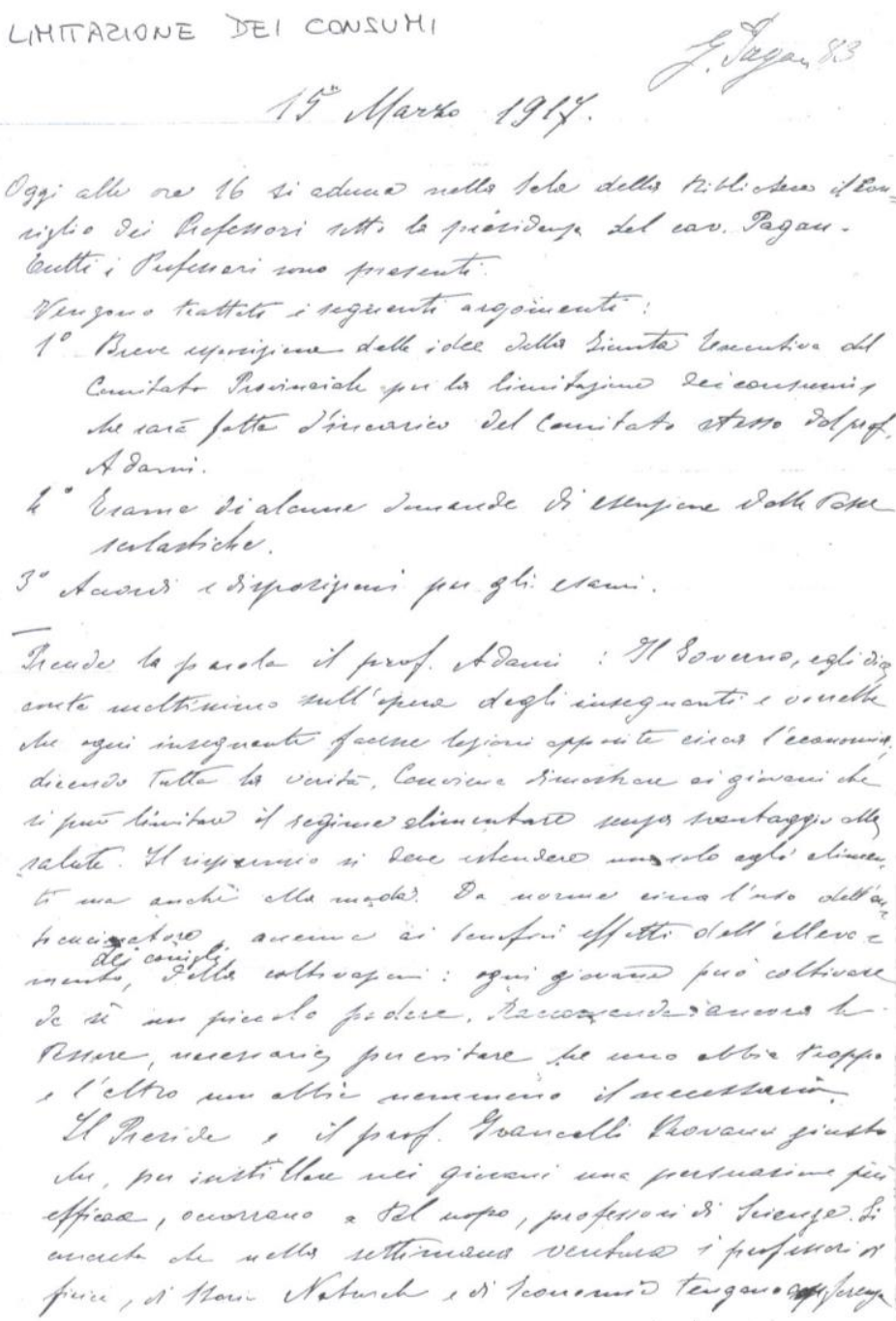

\section{Trascrizione del documento:}

15 Marzo 1917

Oggi alle ore 16 si aduna nella sede della biblioteca il Consiglio dei Professori sotto la presidenza del cav. Pagan.

Tutti i Professori sono presenti

Vengono trattati i seguenti argomenti:

$1^{\circ}$ Breve esposizione delle idee della Giunta Esecutiva del Comitato Provinciale per la limitazione dei consumi, che sarà fatta d'incarico del Comitato stesso dal prof. Adami. [...] Prende la parola il prof. Adami: Il Governo, egli dice, conta moltissimo sull'opera degli insegnanti e vorrebbe che ogni insegnante facesse lezioni apposite circa l'economia, dicendo tutta la verità. Conviene dimostrare ai giovani che si può limitare il regime alimentare senza svantaggio alla salute. II risparmio si deve 
estendere non solo agli alimenti ma anche alla moda. Da norme circa l'uso dell'autocucinatore accenna ai benefici effetti dell'allevamento dei conigli, delle coltivazioni: ogni giovane può coltivare da sé un piccolo podere. II Preside e il prof. Grancelli trovano giusto che, per instillare nei giovani una persuasione più efficace, occorrano a tal uopo, professori di Scienze. Si concorda che nella settimana ventura i professori di fisica, di Storia Naturale e di Economie tengano conferenze in proposito.

(documento tratto dall' Archivio del Liceo Maffei di Verona, Verbali Collegio Docenti, VIT 134 A, p. 83)

\section{Documento 5}

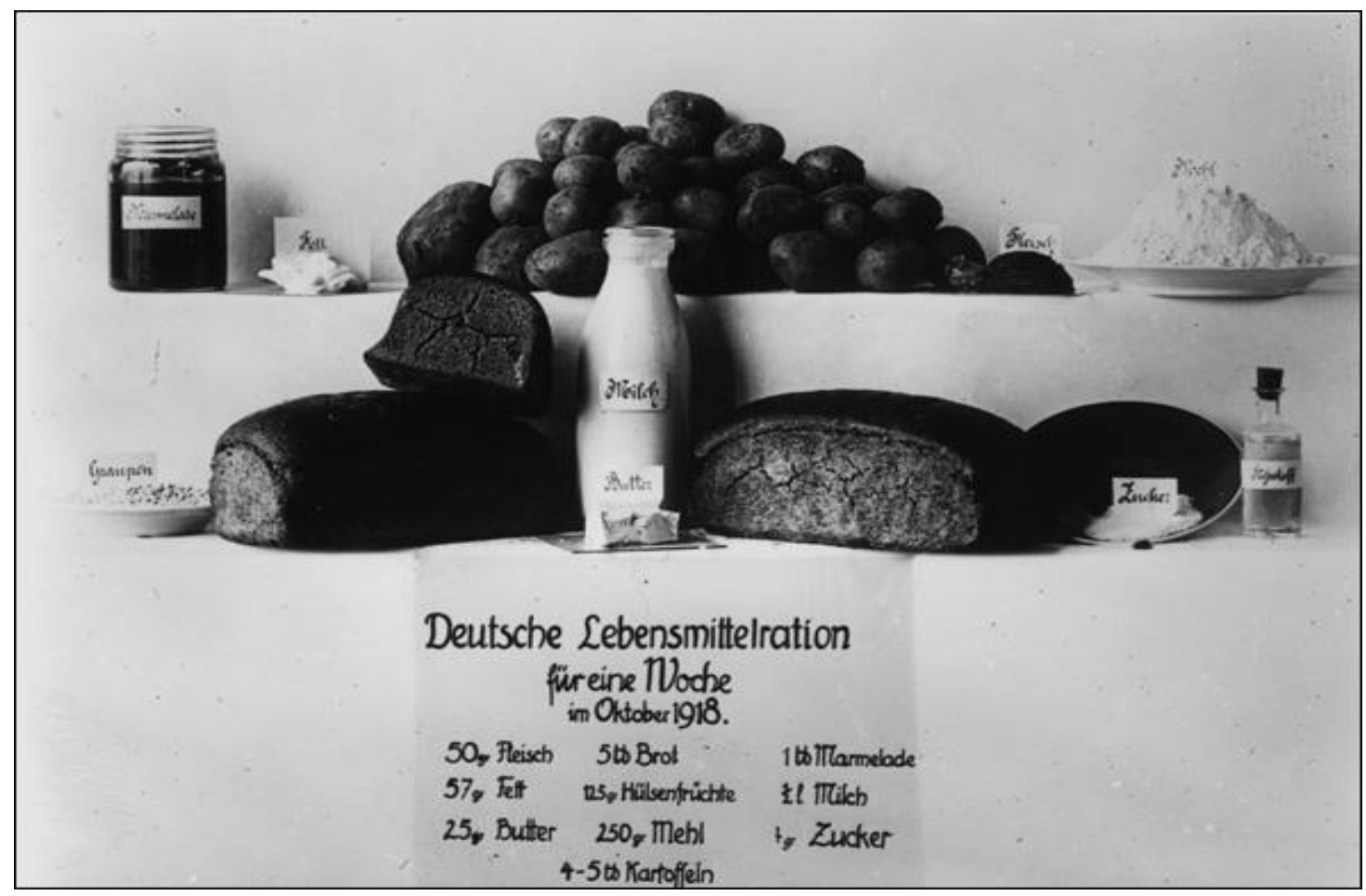

Fotografia della razione alimentare settimanale tedesca nell'ottobre 1918 , conservata presso il (C) Bildarchiv Preußischer Kulturbesitz e riportata sul sito: http://germanhistorydocs.ghidc.org/sub image.cfm?image id=1731 (24.2.2015)

\section{Documento 6}

Nel periodo precedente la guerra, il consumo della carne in Italia - uno dei più bassi in Europa, meno della metà rispetto alla Francia, quasi un quarto rispetto alla Gran Bretagna e alla Germania - era riservato, tra i ceti popolari, a momenti particolari o, comunque, riguardava parti e qualità tra le meno pregiate [...]. Con la guerra, nel passaggio di milioni di italiani, ora soldati, da [una] dieta sostanzialmente vegetariana a una dieta carnea, le cose mutarono notevolmente, né si poteva pretendere che lo sforzo fisico e morale delle truppe impegnate al fronte potesse essere affrontato con un'alimentazione povera di sostanze proteiche e di grassi. 
Inizialmente, la razione carnea giornaliera dei soldati al fronte fu fissata in 375 grammi: in seguito, nel dicembre 1916, anche su consiglio di alcuni fisiologi che ritenevano troppo "lussuosa" la dieta dell'Esercito italiano, la razione passò a 250 grammi, sostituibili, due volte la settimana, con il baccalà. Dopo la disfatta di Caporetto, attribuita da Silvio Crespi, il nuovo responsabile degli Approvvigionamenti e consumi, a una depressione morale causata da scarso nutrimento dell'Esercito, la razione fu portata a 350 grammi, con un aumento nel consumo di carne di circa il $50 \%$, rispetto al periodo prebellico e causando un notevole scompenso nel patrimonio zootecnico nazionale, in particolare quello bovino. Nel dicembre del 1916, insieme alla carne, furono diminuiti anche gli altri generi della dieta giornaliera del soldato al fronte (per il pane, ad esempio, si passò da 750 a 600 grammi), riducendo l'apporto proteico da quasi 4.000 a 3.067 calorie. Nello stesso periodo, dicembre 1916, le razioni francesi fornivano 3.400 calorie, 4.400 quelle inglesi. Nel dicembre 1917, dopo Caporetto, gli aumenti nella razione di carne e pane (da 350 a 700 grammi), il ripristino di 50 grammi di formaggio e l'aumento di 10 grammi di zucchero, portarono la dieta italiana a 3.067 calorie e a giugno 1918 si raggiunsero le 3.580 calorie.

(tratto da: Dentoni M.C., L'alimentazione e l'approvvigionamento alimentare durante il conflitto, in Labanca N. (acd) 2014, Dizionario storico della prima guerra mondiale, Bari: Laterza, pp. 234-235) 


\section{DOSSIER DI LAVORO}

1. Completate la tabella con le informazioni sui diversi alimenti contenute nel documento 1, facendo attenzione alle date di riferimento

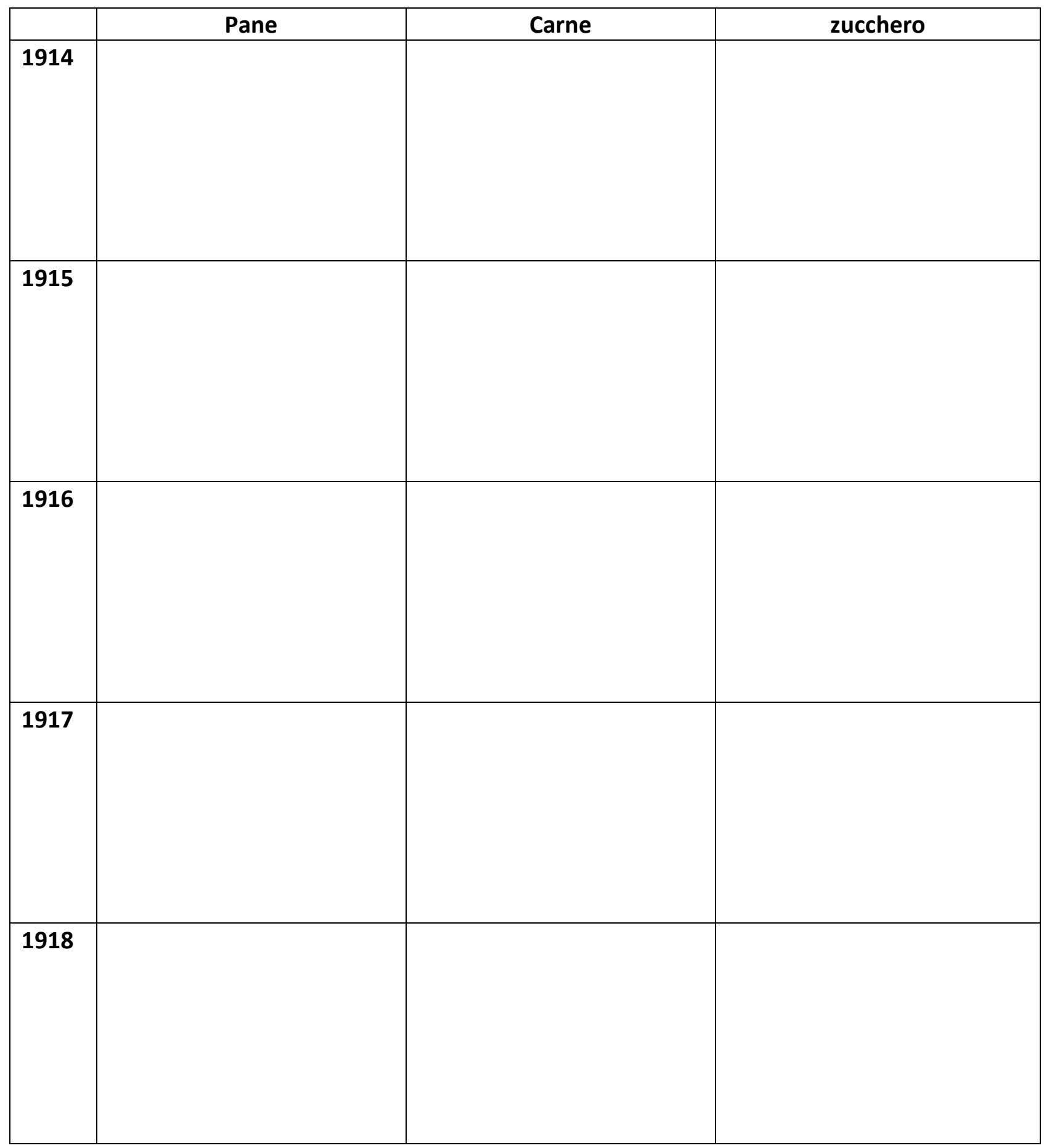


2. Il documento 2 è una lettera spedita da un soldato al fronte alla moglie. Individuate quali delle affermazioni contenute nella lettera riguardano i problemi di alimentazione dei civili e sottolineatele con un colore a vostra scelta. Cercate nel testo iniziale il riferimento a questo problema e sottolineatelo con lo stesso colore.

3. Il documento $\mathbf{2}$ è una lettera spedita da un soldato al fronte alla moglie. Individuate quali delle affermazioni contenute nella lettera riguardano i problemi relativi allo svolgimento dei lavori agricoli nelle campagne e sottolineatele con un colore a vostra scelta. Cercate nel testo iniziale il riferimento a questo problema e sottolineatelo con lo stesso colore.

4. Guardate le fonti riportate nel documento 3: la prima è un volantino, l'altra un articolo del giornale di Verona. Secondo voi, a chi erano rivolte e perché erano state scritte? Aiutatevi cercando riferimenti nel testo iniziale e poi rispondete in massimo 8 righe.

5. Nel testo iniziale si parla della propaganda per la limitazione dei consumi fatta nelle scuole. Trovate la frase che riporta questa informazione, sottolineatela e contrassegnatela con un simbolo a vostra scelta. Poi trovate nel dossier il documento che può provare questa affermazione e contrassegnatelo con lo stesso simbolo. 
6. Osservate con attenzione il documento 5. La fotografia riporta la razione di cibo che un tedesco poteva avere in una settimana nell'ottobre del 1918. Sul cartello leggiamo le quantità degli alimenti concessi con la tessera annonaria:

$50 \mathrm{~g}$ di carne, $57 \mathrm{~g}$ di grasso, $25 \mathrm{~g}$ di burro, $2,5 \mathrm{~kg}$ di pane, $125 \mathrm{~g}$ di legumi, $250 \mathrm{~g}$ di farina, 2-2,5 $\mathrm{kg}$ di patate, $500 \mathrm{~g}$ di marmellata, $1 / 2$ I di latte e $1 \mathrm{~g}$ di zucchero.

Inserite i dati nella tabella qui sotto. Nell'ultima colonna sono già indicate le calorie corrispondenti alla razione. Calcolate il numero di calorie di cui un tedesco poteva disporre. La razione era settimanale, quindi quante calorie aveva a disposizione al giorno?

CALORIE OGNI 100 GRAMMI OPPURE OGNI 10 CL PER LE TIPOLOGIE DI ALIMENTI INDICATI

\begin{tabular}{|l|c|c|c|}
\hline ALIMENTO & $\begin{array}{c}\text { CALORIE/100 g } \\
\mathbf{0} \mathbf{1 0} \mathbf{~ c l}\end{array}$ & $\begin{array}{c}\text { QUANTITÁ DI ALIMENTO } \\
\text { DELLA RAZIONE } \\
\text { SETTIMANALE }\end{array}$ & $\begin{array}{c}\text { CALORIE IN BASE ALLA } \\
\text { RAZIONE }\end{array}$ \\
\hline LATTE & 63 CALORIE & & 315 \\
\hline PATATE & 83 CALORIE & & 2075 \\
\hline MARMELLATA & 284 CALORIE & & 1420 \\
\hline $\begin{array}{l}\text { LEGUMI } \\
\text { (FAGIOLI) }\end{array}$ & 330 CALORIE & & 412,5 \\
\hline BURRO & 750 CALORIE & & 5750 \\
\hline PANE & 230 CALORIE & & 507,87 \\
\hline $\begin{array}{l}\text { GRASSO } \\
\text { (LARDO) }\end{array}$ & 891 CALORIE & & 797,5 \\
\hline FARINA & 310 CALORIE & & 3,92 \\
\hline ZUCCHERO & 392 CALORIE & & 65 \\
\hline $\begin{array}{l}\text { CARNE } \\
\text { BOVINA }\end{array}$ & 130 CALORIE & & \\
\hline & & Totale calorie & \\
\hline & & Calorie giornaliere & \\
\hline
\end{tabular}


7. Il documento 6 riporta la quantità di calorie di cui potevano disporre i soldati al fronte: ricopiate le informazioni nella tabella.

\begin{tabular}{|l|l|l|l|}
\hline \multirow{2}{*}{1914} & Soldati italiani & Quantità di calorie & \\
\hline & & & \\
\hline 1915 & & & \\
\hline 1916 & & & \\
\hline 1917 & & & \\
\hline 1918 & & & \\
\hline
\end{tabular}

In quale nazione i soldati potevano disporre del maggior numero di calorie?

Confrontate questi dati con le calorie a disposizione dei tedeschi (esercizio precedente).

Secondo voi, l'alimentazione dei soldati può aver influito sull'esito della guerra?

Trovate nel testo iniziale la frase che può fornire la risposta a questa domanda e sottolineatela. 


\section{BIBLIOGRAFIA:}

Beltrame Menini L. 2014, Ta-pum. Lettere dal fronte, Padova: F.lli Corradin Editori.

Capatti A., De Bernardi A., Varmi A. (acd) 1998, Storia d'Italia, Annali 13. L'alimentazione, Torino: Einaudi.

Dentoni M.C. 1995, Annona e consenso in Italia 1914-1919, Milano: Angeli.

Dentoni M.C., L'alimentazione e l'approvvigionamento alimentare durante il conflitto, in Labanca N. (acd) 2014, Dizionario storico della prima guerra mondiale, Roma-Bari: Laterza, pp. 230-238.

Flandrin J-L., Montanari M. 1997, Storia dell'alimentazione, Roma-Bari: Laterza.

Gibelli A. 1999, La grande guerra degli italiani 1915-1918, Milano: Sansoni.

Luciani E. 2002, Giornalisti in trincea. L'informazione durante la Grande Guerra in una città di retrovia, Verona: Gemma Editco.

Perin A. 2005, La fame aguzza l'ingegno, Milano: Elèuthera.

Procacci G., L'Italia nella Grande Guerra, in Sabatucci G., Vidotto V. (acd) 1997, Storia d'Italia, vol. IV Guerre e fascismo 1914-1943, Roma-Bari: Laterza, pp. 3-99.

\section{Sitografia:}

Istituto Parri di Bologna:

https://guerrainfame.it/progetto

Motore di ricerca europeo sulla Grande Guerra:

http://www.europeana1914-1918.eu/en

Siti con contenuti supervisionati da docenti universitari:

http://www.centoannigrandeguerra.it/ (prof. De Luna)

http://www.lagrandeguerrapiu100.it/ (prof. Corni) 